\title{
Boundary Layer Flow Of Nanofluid Past An Inclined Stretching Sheet
}

\author{
JULIE ANDREWS
}

\begin{abstract}
Nanofluids, in which nano-sized particles (typically less than 100 nanometers) suspended in liquids, have emerged as a potential candidate for the design of heat transfer fluids. The main goal of nanofluids is to achieve the highest possible thermal properties at the smallest possible concentrations by uniform dispersion and stable suspension of nanoparticles in host fluids. This paper also includes diverse applications of nanofluids. Along with the cooling applications, there are tribological and medical applications for nanofluids. Nanofluids could be utilized for a wide variety of applications in day today life. The problem of laminar fluid flow which results from an inclined stretching flat surface in nanofluid has been investigated numerically in this paper. The model used here for the considers the effects of Brownian motion and thermophoresis. A similarity solution is presented which depends on the Prandtl number Pr, Lewis number Le, Brownian number $\mathrm{Nb}$ and thermophoresis number Nt. The coupled partial differential equations governing the flow are transformed into nonlinear boundary layer equations, which are then solved using the Nachtsheim-Swigert Shooting iteration technique along with the fourth order Runga Kutta method. The variation of the Nusselt and Sherwood numbers with $\mathrm{Nb}, \mathrm{Nt}, \mathrm{Pr}$, Le, Gr and Ge for various values of angle of inclinations $\alpha$ is presented in graphical forms.
\end{abstract}

Keywords - Boundary-layer, nanofluid, inclined stretching sheet, similarity solution

\section{Introduction}

The boundary layer flows over a stretching surface have abundant applications in engineering. Some of the specific examples of such problems are continuous casting, cooling of electronic chips, crystal growing, polymer melts and solutions and so on. Nanotechnology has been widely used in industry since materials with sizes of nanometers possess unique physical and chemical properties. Nano-scale particle added fluids are called as nanofluid, which is firstly utilized by Choi [1]. Nano fluids represent an innovative way to increase thermal conductivity and, therefore, heat transfer. Unlike heat transfer in conventional fluids, the exceptionally high thermal conductivity of nanofluids provides for enhanced heat transfer rates, a unique feature of nanofluids. Advances in device miniaturization have necessitated heat transfer systems that are small in size, light mass, and high-performance [2]. Khan

Julie Andrews(Author)

St. Thomas' College(Autonomous), Thrissur, Kerala India and Pop [3] have studied the problem of laminar fluid flow which results from the stretching of a flat surface in a nanofluid and investigated it numerically.

\section{A. Applications}

Nanofluids find most of their applications in thermal management of industrial and consumer products as efficient cooling is vital for realizing the functions and long-term reliability of the same. There are a large number of tribological and medical applications for nanofluids. Nanofluids are promising coolants of the next generation as they could challenge the ordinary existing coolants in heat transfer

The cooling applications of nanofluids include Crystal Silicon Mirror Cooling, Electronics Cooling, Vehicle Cooling, Transformer Cooling, Space and Nuclear systems Cooling, Defense applications and so on. Nanofluid technology can surely help to develop better oils and lubricants. Recently the use of nanoparticles in lubricants to enhance tribological properties of lubricants, such as load-carrying capacity, antiwear and friction-reducing properties between moving mechanical components has developed as the nanofluid research activity. Nanofluids are now being developed for medical applications, including cancer therapy. As traditional cancer treatment methods have significant side effects, Ironbased nanoparticles can be used as delivery vehicles for drugs or radiation without damaging nearby healthy tissue by guiding the particles up the bloodstream to a tumour with magnets.

\section{II.}

\section{Mathematical}

\section{Formulation}

The present problem considers the steady, nonlinear twodimensional, mixed convective boundary layer flow of a viscous, incompressible nanofluid past an inclined stretching surface with an angle of inclination $\alpha$ with the horizontal and stretching with the linear velocity $\mathrm{u}_{\mathrm{w}}(\mathrm{x})=\mathrm{ax}$, where $\mathrm{a}$ is a constant and $\mathrm{x}$ is the coordinate measured along the stretching surface. The sheet is stretched keeping the origin fixed when $\alpha$ is $0^{\circ}$ and the variations are made accordingly with the change in inclination angles. The Cartesian coordinates $(x, y)$ are chosen such that $\mathrm{x}$-axis is chosen along the stretching sheet and $y$-axis is chosen perpendicular to it. It is assumed that at the stretching surface, the temperature $\mathrm{T}$ and the nanoparticle 
fraction $C$ take constant values $T_{w}$ and $C_{w}$, respectively. The ambient values, attained as $\mathrm{y}$ tends to infinity, of $\mathrm{T}$ and $\mathrm{C}$ are denoted by $\mathrm{T}_{\infty}$ and $\mathrm{C}_{\infty}$, respectively. Here in this paper Copper-water nanofluid is the nanofluid chosen with water as the base fluid with $\operatorname{Pr}=7.02$ considering base temperature as $293 \mathrm{~K}$. Solid spherical copper nano particles of $100 \mathrm{~nm}$ diameter mixed with water, has been considered as the nanofluid.

\section{A. Governing Equations}

The basic steady conservation of mass, momentum, thermal energy and nanoparticles equations for nanofluids can be written in Cartesian coordinates $\mathrm{x}$ and $\mathrm{y}$ as, see Kuznetsov and Nield [4,5].The governing Prandtl boundary layer equations for the steady two dimensional laminar nanofluid flow over the inclined stretching surface are as follows:

$$
\begin{aligned}
& \frac{\partial u}{\partial x}+\frac{\partial v}{\partial y}=0 \\
& u \frac{\partial u}{\partial x}+v \frac{\partial u}{\partial y}=g \beta_{n f}\left(T-T_{\infty}\right) \sin \alpha+g \beta_{n f}^{*}\left(C-C_{\infty}\right) \sin \alpha \\
& -\frac{1}{\rho_{n f}} \frac{\partial p}{\partial x}+v_{n f}\left(\frac{\partial^{2} u}{\partial y^{2}}\right) \\
& 0=g \beta_{n f}\left(T-T_{\infty}\right) \cos \alpha+g \beta_{n f}^{*}\left(C-C_{\infty}\right) \cos \alpha-\frac{1}{\rho_{n f}} \frac{\partial p}{\partial y} \\
& u \frac{\partial T}{\partial x}+v \frac{\partial T}{\partial y}=\alpha_{n f}\left(\frac{\partial^{2} T}{\partial y^{2}}\right) \\
& +\varepsilon\left[D_{B}\left(\frac{\partial C}{\partial y} \frac{\partial T}{\partial y}\right)+\frac{D_{T}}{T_{\infty}}\left(\frac{\partial T}{\partial y}\right)^{2}\right] \\
& u \frac{\partial C}{\partial x}+v \frac{\partial C}{\partial y}=D_{B}\left(\frac{\partial^{2} C}{\partial y^{2}}\right)+\frac{D_{T}}{T_{\infty}}\left(\frac{\partial^{2} T}{\partial y^{2}}\right)
\end{aligned}
$$

Moreover, the boundary conditions for the velocity and temperature of this problem are:

$$
\begin{aligned}
& u=u_{w}(x)=a x, v=0, T=T_{W}, C=C_{W} \text { at } y=0 \\
& u=0, T=T_{\infty}, C=C_{\infty} \text { as } y \rightarrow \infty
\end{aligned}
$$

Here, $\mathrm{u}$ and $\mathrm{v}$ are the velocity components in $\mathrm{x}$ and $\mathrm{y}$ directions respectively, $\mathrm{p}$ is the perturbed fluid pressure, $\mathrm{T}$ is taken as the temperature, $v_{n f}$ is the kinematic viscosity of the nanofluid, $\mathrm{k}_{\mathrm{nf}}$ is the thermal conductivity, $\left(\mathrm{c}_{\mathrm{p}}\right)_{\mathrm{nf}}$ is the specific heat, $\alpha_{\mathrm{nf}}$ is the thermal diffusivity, $\beta_{n f}$ is the coefficient of thermal expansion of the nanofluid, $\beta_{n f}^{*}$ is the coefficient of volumetric coefficient of expansion with concentration, $\mathrm{C}$ is the nanoparticle volume fraction and $\rho_{\mathrm{nf}}$ is the density of the nanofluid respectively. Here, a is a positive constant, $D_{B}$ is the Brownian diffusion coefficient, $D_{T}$ is the thermophoretic diffusion coefficient and $\varepsilon=\frac{(\rho c)_{p}}{\left(\rho c_{p}\right)}$ is the ratio between the effective heat capacity of the nanoparticle material and heat capacity of the fluid with $\rho$ being the density. Thus the governing Prandtl boundary layer equations for the steady two dimensional laminar nanofluid flow over an inclined stretching surface when $0^{\circ} \leq \alpha \leq 60^{\circ}$ take the following form:

$$
\begin{aligned}
& \frac{\partial u}{\partial x}+\frac{\partial v}{\partial y}=0 \\
& u \frac{\partial u}{\partial x}+v \frac{\partial u}{\partial y}=v_{n f} \frac{\partial^{2} u}{\partial y^{2}}+g \beta_{n f}\left(T-T_{\infty}\right) \sin \alpha+ \\
& g \beta_{n f}^{*}\left(C-C_{\infty}\right) \sin \alpha \\
& u \frac{\partial T}{\partial x}+v \frac{\partial T}{\partial y}=\alpha_{n f}\left(\frac{\partial^{2} T}{\partial y^{2}}\right)+\varepsilon\left[D_{B}\left(\frac{\partial C}{\partial y} \frac{\partial T}{\partial y}\right)+\frac{D_{T}}{T_{\infty}}\left(\frac{\partial T}{\partial y}\right)^{2}\right] \\
& u \frac{\partial C}{\partial x}+v \frac{\partial C}{\partial y}=D_{B}\left(\frac{\partial^{2} C}{\partial y^{2}}\right)+\frac{D_{T}}{T_{\infty}}\left(\frac{\partial^{2} T}{\partial y^{2}}\right)
\end{aligned}
$$

III.

\section{Method Of Solution}

The following dimensionless variables are introduced in order to seek the solution of the problem:

$$
\begin{aligned}
& \psi(x, y)=\left(a_{n f}\right)^{1 / 2} x f(\eta) \quad, \eta=y\left(\frac{a}{v_{n f}}\right)^{1 / 2}, \\
& \theta=\frac{T-T_{\infty}}{T-T}, \quad \phi=\frac{C-C_{\infty}}{C_{W}-C_{\infty}}
\end{aligned}
$$

where $\psi(x, y)$ is the stream function and velocity components can be expressed as

$$
u=u_{w} f^{\prime}(\eta)
$$

$$
v=-\left(a v_{n f}\right)^{1 / 2} f(\eta)
$$


Accordingly, momentum, energy and concentration equations together with the boundary conditions, can be written as

$$
\begin{aligned}
& f^{\prime \prime \prime}+f f^{\prime \prime}-\left(f^{\prime}\right)^{2}+\lambda \theta \sin \alpha+\lambda * \phi \sin \alpha=0 \\
& \frac{1}{(\operatorname{Pr})}{ }_{n f} \theta^{\prime \prime}+f \theta^{\prime}+N_{b} \phi^{\prime} \theta^{\prime}+N_{t}\left(\theta^{\prime}\right)^{2}=0 \\
& \phi^{\prime \prime}+(S c)_{n f} f \phi^{\prime}+\frac{N_{t}}{N_{b}} \theta^{\prime \prime}=0
\end{aligned}
$$

with the corresponding boundary conditions $f(0)=0, f^{\prime}(0)=1, \theta(0)=1, \phi(0)=1$

$$
f^{\prime}(\infty)=0, \theta(\infty)=0, \phi(\infty)=0
$$

Here primes denote differentiation with respect to $\eta$ and $v_{\mathrm{nf}}$ and $\alpha_{\mathrm{nf}}$ are the kinematic viscosity and thermal diffusivity of the nanofluid respectively. Also, $(\mathrm{Pr})_{\mathrm{nf}},(\mathrm{Sc})_{\mathrm{nf}}, \mathrm{N}_{\mathrm{b}}$ and $\mathrm{N}_{\mathrm{t}}$ denote Prandtl number, Schmidt number, Brownian motion parameter and thermophoresis parameter respectively. Here, $\left(\operatorname{Re}_{\mathrm{x}}\right)_{\mathrm{nf}}$ is the local Reynolds number, $\lambda$ is the mixed convection parameter, $\lambda^{*}$ is the modified mixed convection parameter, $\left(\mathrm{Gr}_{\mathrm{x}}\right)_{\mathrm{nf}}$ is the local Grashof number and $\left(\mathrm{Gc}_{\mathrm{x}}\right)_{\mathrm{nf}}$ is the modified local Grashof number of the nanofluid.

\section{A. Numerical Analysis}

The set of nonlinear differential equations subject to the boundary conditions constitute the nonlinear boundary value problem. The above boundary value problem is converted into an initial value problem by shooting method. As the ordinary methods fail in order to solve the system of transformed equations together with the asymptotic boundary conditions, a special iteration technique is used. Equations are solved numerically subject to using Fourth-Order Runge-Kutta based shooting method along with Nachtsheim-Swigert iteration scheme for satisfaction of asymptotic boundary conditions. Initial guesses for the values of $f^{\prime \prime}(0)$ and $\theta^{\prime}(0)$ are made to initiate the shooting process and these initial guesses are made taking into account of convergency and numerical results are obtained for several values of the physical parameter $(\operatorname{Pr})_{n f}$.

Concerning this study, quantities which are of interest are the non-dimensional skin friction coefficient $C_{f}$, Nusselt number $N u$ and Sherwood number $S h$ which are defined as

$$
\begin{gathered}
C_{f}=\frac{\tau_{w}}{\rho_{f} U_{\infty} 2}, \text { where } \tau_{w}=\mu_{n f}\left(\frac{\partial u}{\partial y}\right)_{y=0} \\
N u=\frac{x q_{w}}{k_{f}\left(T_{w}-T_{\infty}\right)}, \text { where } q_{w}=-k_{n f}\left(\frac{\partial T}{\partial y}\right)_{y=0} \\
S h=\frac{x q_{m}}{D_{B}\left(C_{w}-C_{\infty}\right)}, \quad q_{m}=-D_{B}\left(\frac{\partial C}{\partial y}\right)_{y=0}
\end{gathered}
$$

where $q_{w}$ and $q_{m}$ are the wall heat flux and mass flux respectively. Kuznetsov and Nield (2009; 2010) referred

$$
\begin{aligned}
& N u\left(\operatorname{Re}_{x}\right)_{n f^{-1 / 2} \text { and }} \\
& \operatorname{Sh}\left(\operatorname{Re}_{x}\right)_{n f}^{-1 / 2} \text { as reduced Nusselt number } \\
& \text { Nur }=-\frac{k_{n f}}{k_{f}} \theta^{\prime}(0) \text { and } \\
& \text { reduced Sherwood number }
\end{aligned}
$$$$
\mathrm{Shr}=-\frac{\left(D_{B}\right)_{n f}}{\left(D_{B}\right) f} \phi^{\prime}(0) \text { respectively. }
$$

IV.

\section{Results and}

\section{Discussions}

The values of different parameters considered here are as follows: $\boldsymbol{\phi}=0.00,0.01,0.05,0.10, \mathrm{Nt}=0.1,0.2,0.3,0.4,0.5, \alpha$ $=0^{\circ}, 30^{\circ}, 45^{\circ}$ and $60^{\circ}$ and $\operatorname{Pr}=6.06$ (copper water nanofluid ) The following values are fixed : $\mathrm{Le}=10, \mathrm{Nb}=0.1, \mathrm{Gr}=10$, $\mathrm{Gc}=10$.

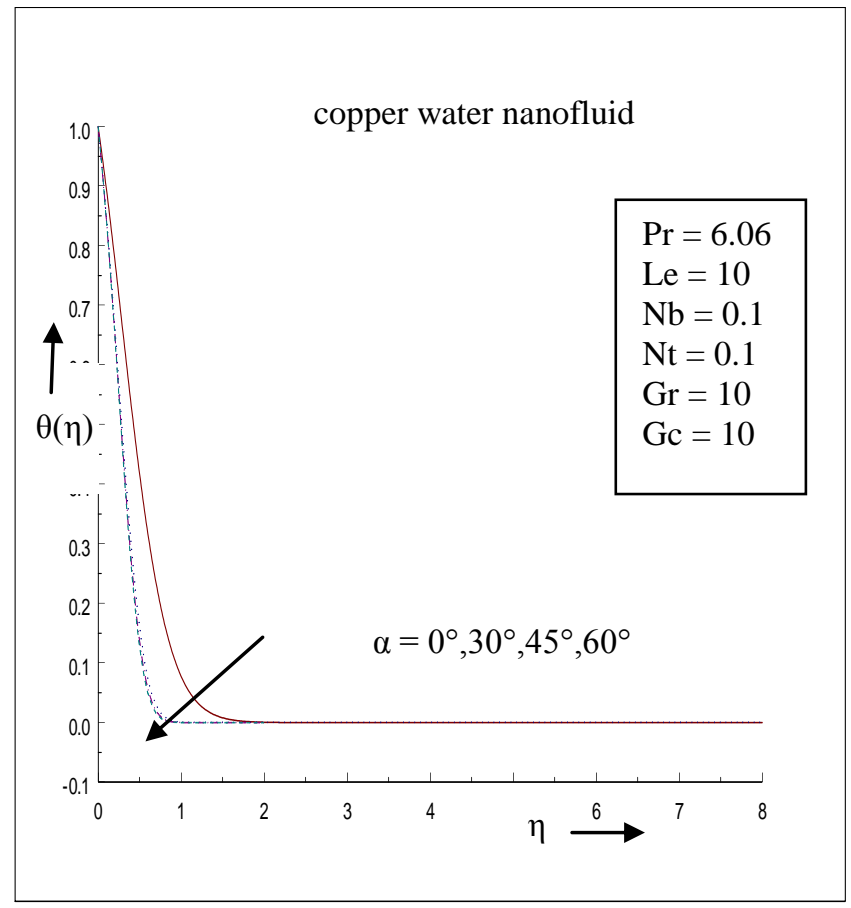

Figure 1. Effect of inclination angle on temperature distribution for specified parameters for copper water nanofluid 
Proc. of the Fifth International Conference on Advances in Mechanical and Robotics Engineering - AMRE 2017. Copyright $\odot$ Institute of Research Engineers and Doctors. All rights reserved.

ISBN: 978-1-63248-123-8 doi: 10.15224/ 978-1-63248-123-8-38

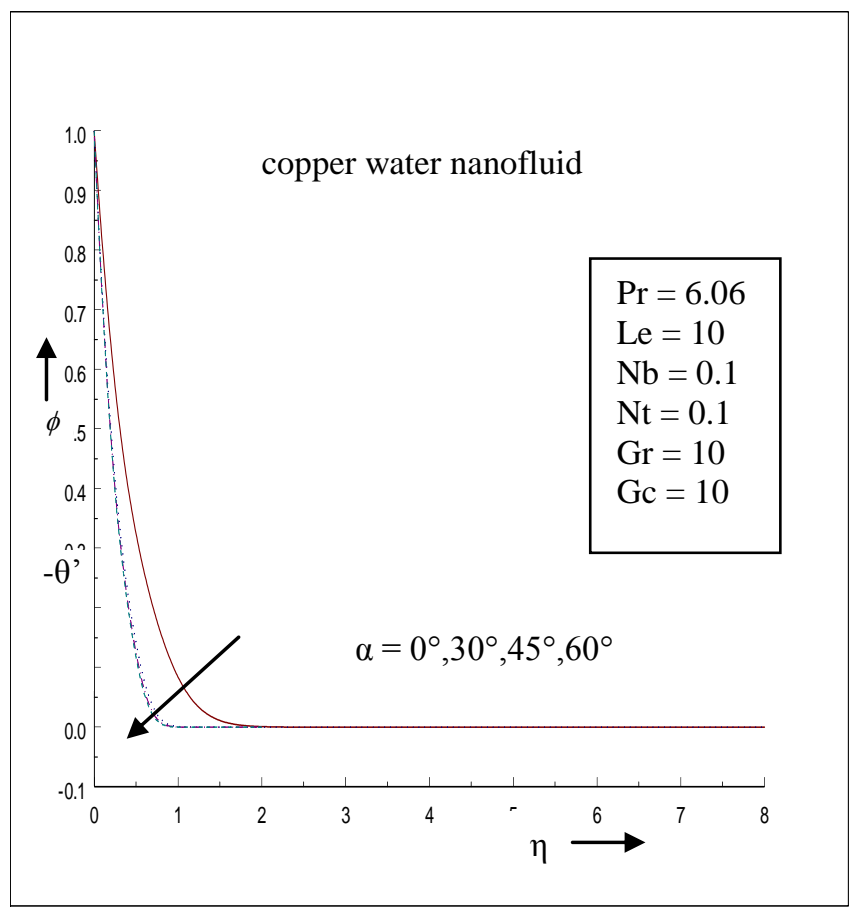

Figure 2. Effect of inclination angle on concentration distribution for specified parameters for copper water nanofluid

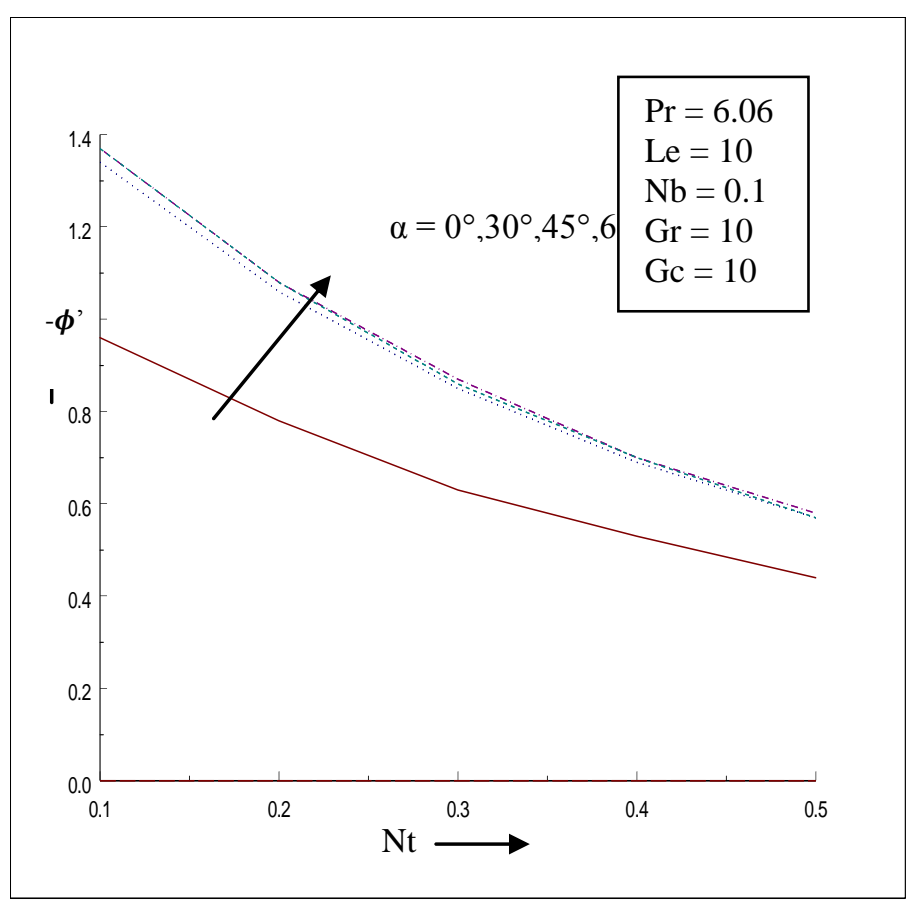

Figure. 3.Effects of inclination angles on dimensionless heat transfer rates

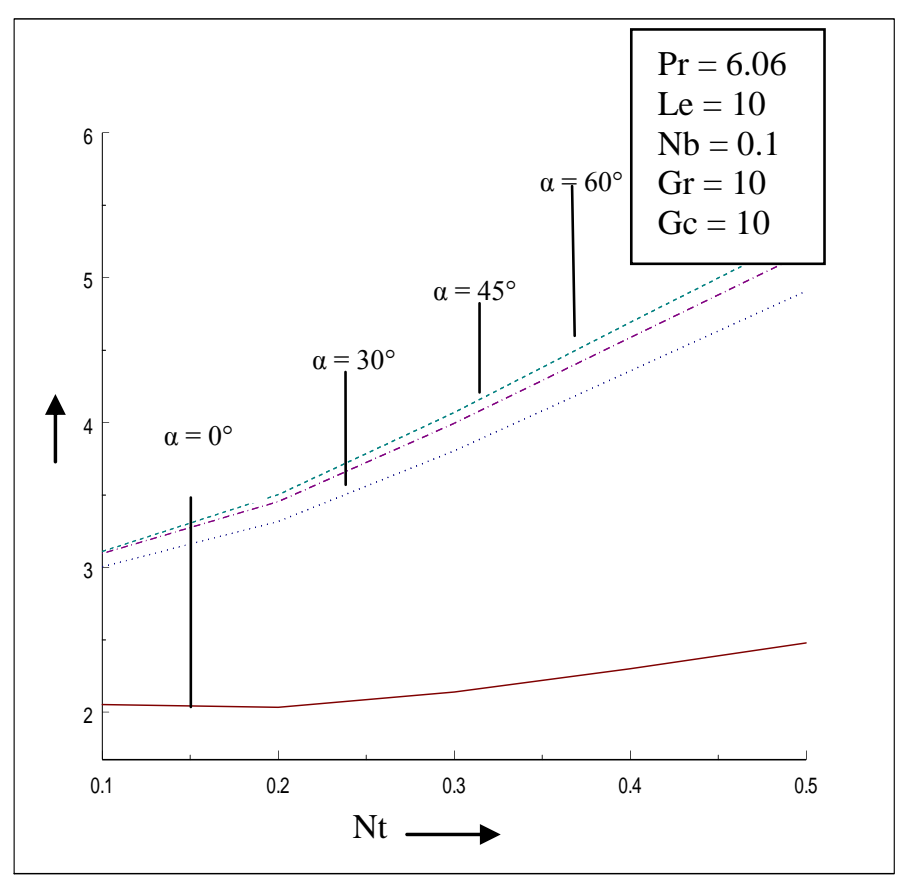

Figure. 4.Effects of inclination angles on dimensionless mass transfer rates

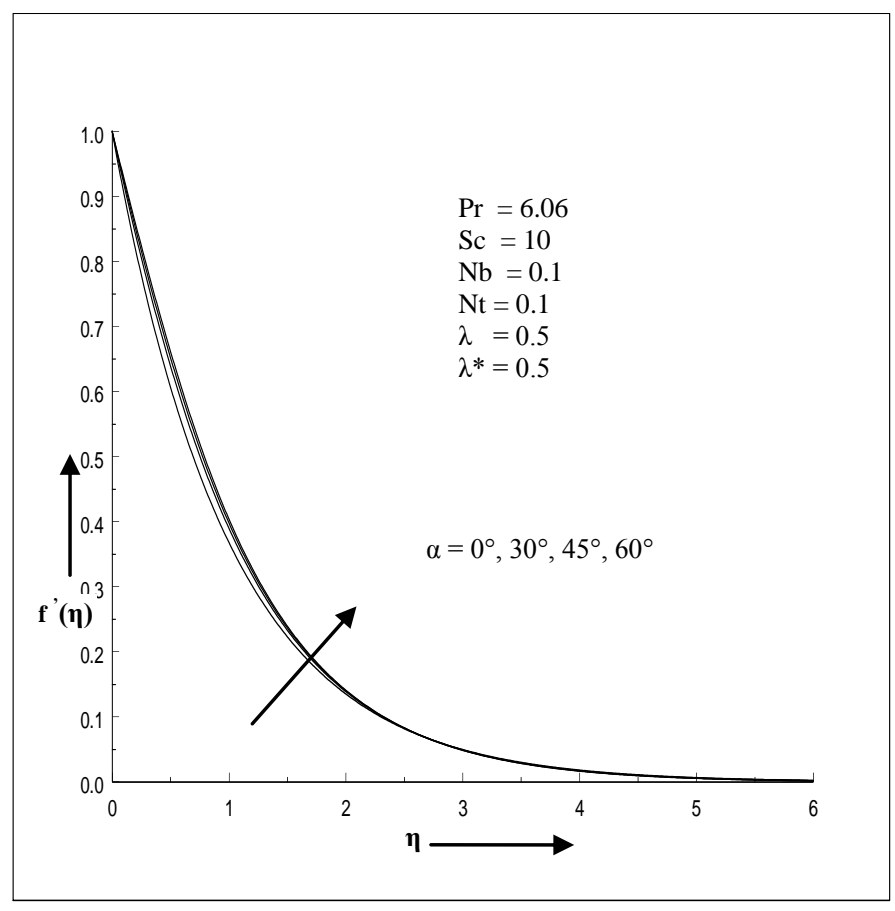

Figure.5 Effect of inclination angle over dimensionless velocity 


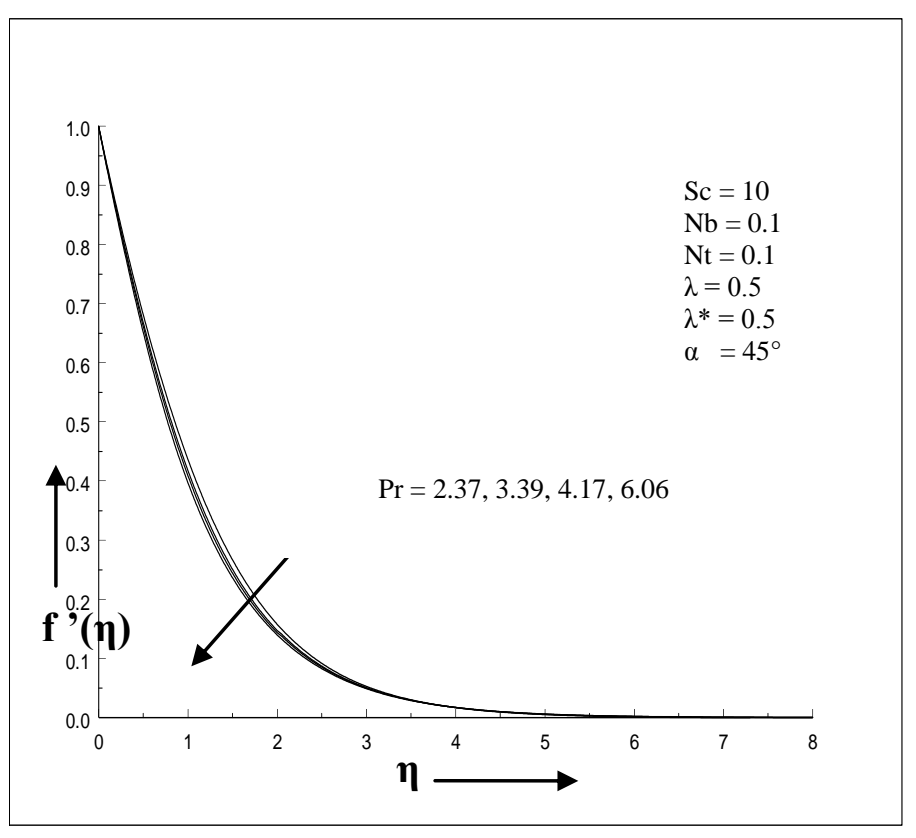

Figure.6 Effect of $\operatorname{Pr}$ over dimensionless velocity with specified parameters

Fig.1 displays the effect of inclination angle $\alpha=$ $0^{\circ}, 30^{\circ}, 45^{\circ}, 60^{\circ}$ on temperature distribution for specified parameters. When $\alpha$ increases, temperature decreases. From Fig.2. it is seen that as $\alpha$ increases, concentration decreases. In Fig. 3, as $\alpha$ increases, dimensionless heat transfer rate also increases. It is shown in Fig.4 that as $\alpha$ increases, dimensionless concentration rate also increases for the same fixed values of Pr, Le,Nb,Nt,Gr and Gc. Fig.5 and Fig.6 displays the effect of angle of inclinations and $\operatorname{Pr}$ on dimensionless velocity with specified parameters.

\section{A. Conclusion}

For copper-water nanofluid, as the inclination angle $\alpha$ increases, dimensionless temperature and dimensionless concentration decrease with increasing $\alpha$.As the inclination angle $\alpha$ increases, reduced Nusselt number Nur and reduced Sherwood number Shr increases respectively. When the angle of inclination increases, dimensionless velocity increases. As Pr increases, dimensionless velocity decreases. As nanofluids are highly efficient, in the field of industry and medicine, they could be used as coolants required for so many purposes. Nanofluids will be the smart coolants in the coming era as using them as coolants, they could be highly effective as the quality of the coolant improves the heat transfer and the size of the instruments used could be miniaturized.

\section{References}

[1] S.U.S. Choi, Enhancing thermal conductivity of fluids with nanoparticles, The Proceedings of the 1995 ASME International Mechanical Engineering Congress and Exposition, San Francisco, USA, ASME, FED 231/MD 66, 1995, pp. 99-105.

[2] R S R Gorla, A J Chamkha and A M Rashad.Mixed convective boundary layer flow over a vertical wedge embedded in a porous medium saturated with a nanofluid: Natural Convection Dominated Regime, Nanoscale Research Letters 2011, 6:207.

[3] W.A Khan and I Pop. "Boundary-Layer Flow of a Nanofluid Past a Stretching Sheet," International Journal of Heat and Mass Transfer, Vol. 53, 2010, pp. $2477-2483$.

[4] A.V Kuznetsov and D.A Nield, "Natural Convective Boundary-layer Flow of a Nanofluid Past a Vertical Plate," Int. J. of Thermal Sciences, Vol. 49, 2010, pp. $243-247$.

[5] A.V Kuznetsov and D.A Nield,., "The Cheng-Minkowycz Problem for Natural Convective Boundary-layer Flow in a Porous Medium Saturated by a Nanofluid," In. J. of Heat and Mass Transfer, Vol. 52, 2009, pp. 5792 - 5795.

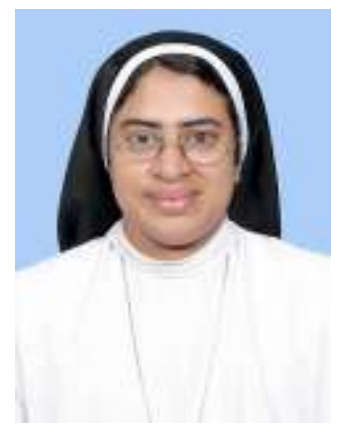

[Nanofluids are promising coolants of the next generation as they could challenge the ordinary existing coolants in heat transfer.] 